\title{
Knowledge-Based Systems
}

\author{
Analysis of pattern recognition and dimensionality reduction techniques for odor \\ biometrics \\ Irene Rodriguez-Lujan, Gonzalo Bailador, Carmen Sanchez-Avila, Ana Herrero, \\ Guillermo Vidal-de-Miguel
}

\begin{abstract}
In this paper, we analyze the performance of several well-known pattern recognition and dimensionality reduction techniques when applied to mass-spectrometry data for odor biometric identification. Moti-vated by the successful results of previous works capturing the odor from other parts of the body, this work attempts to evaluate the feasibility of identifying people by the odor emanated from the hands. By formulating this task according to a machine learning scheme, the problem is identified with a small-sample-size supervised classification problem in which the input data is formed by mass spectro-grams from the hand odor of 13 subjects captured in different sessions. The high dimensionality of the data makes it necessary to apply feature selection and extraction techniques together with a simple clas-sifier in order to improve the generalization capabilities of the model. Our experimental results achieve recognition rates over $85 \%$ which reveals that there exists discriminatory information in the hand odor and points at body odor as a promising biometric identifier.
\end{abstract}

\section{Introduction}

According to the United Nations World Tourism Organization (UNWTO), more than a billion of tourists traveled to other countries during 2012, which marks a new record for international tourism. ${ }^{2}$ This trend supposes that the authorities must make a huge effort in controlling the access at the border checkpoints in order to prevent irregular immigration or to detect wanted persons. Although new electronic passports are more difficult to forge [28], it seems appropriate to introduce some biometric techniques which allow verifying the identity of the subjects. On the one hand, well-accepted techniques, such as face recognition, have low accuracies. On the other hand, techniques based on fingerprint [35] and iris [65] provide higher accuracies by the cost of the possible reticence of the subjects to offer these personal biometric features [25]. Advances in technology have made possible the advent of sensors capturing the body odor, so non-invasive biometric techniques based on this information are being developed [17].

The human identification from body odor is not a new idea, bloodhounds have been used to identify people for more than a century by the police. The well-known ability of these specially trained dogs to follow tracks of a person from just an odor sample shows the viability of using the body odor as biometric identifier $[51,26]$. These animals are so accurate that some studies have shown that they are able to guess the author of a bomb with a success of $60 \%$, even with only a small fragment of the original device [53]. This ability has also been observed in humans but in a lesser extent. For example, the study [32] indicates that some people are able to identify the odor of their relatives.

Identification from body odor is not a trivial task since the scent can vary considerably by diverse factors. Curran et al. [9] propose to classify the body odor in three different categories depending on the permanence of the odor. The primary odor is our individual fragrance generated by our body. The relation among the chemicals compounds that produce this scent remains constant over time even with changes in the diet or other transitory factors. Some studies $[9,31]$ suggest that this primary odor depends on genetic factors thus, these compounds allow people identification. Furthermore, this genetic propensity by every individual to produce specific compounds has been reported even in studies with monozygotic twins [31], in which high correlations between the compounds of both siblings are found. The compounds belonging 
to secondary odor are also stable over time but are produced by a specific diet or other internal factors. For example, the emotional mood, such as a period of depression, may affect our body odor [7]. In women, the different phases of the menstrual cycle have shown to change the body odor [24]. What is more, some medical works have identified some compounds in the odor that indicate the presence of certain diseases as skin cancer [11]. Finally, tertiary odor compounds are due to the external application of different cosmetics as lotions, soaps, perfumes, etc. and therefore, they are not produced by our own body.

In addition to this intra-personal variability, some studies report some differences in the odor generated by people of diverse ethnic groups as European or Japanese [52]. The relation between the concentration of some compounds and the gender of the person is also analyzed in $[12,44]$. Although some studies found some differences in the odor due to the aging, these differences may be caused by the specific diet of the studied population [16]. Finally, other environmental factors as the humidity or the temperature of the acquisition room have shown to affect considerably the composition of our body odor [63].

Previous studies $[2,3]$ to identify those compounds of our scent that attract yellow fever mosquitoes have found many common compounds in human body odor. Our odor is composed of chemicals known as volatiles which include hydrocarbons, alcohols, carboxylic acids, ketones and aldehydes [50]. In particular, some works analyzing axillary odor $[61,62,42]$ identify these compounds as mainly linear chains $C_{6}-C_{10}$, normal, branched, hydroxy- and unsaturated acids. These authors also suggest that the main contributor to human body odor is acid (E)-3-metil-2-hexanoic, although other compounds as acids 2-metil $C_{6}-C_{10}$ and acids 4-etil $C_{5}-C_{11}$ are also abundant.

The body odor production is a complex process that has not been still completely understood. Most of the odor is thought to be generated by the epithelial cells detached from our epidermis, body secretions and the bacteria fed with them [9]. The skin surface has about 2 billion of cells and 1/30 of them are daily detached, i.e., the human body emits about 667 cells per second. Each cell is usually accompanied by about four bacteria and secretions from apocrine, eccrine and sebaceous glands. The conjunction of these three components is considered characteristic of each person [9]. Nevertheless, other works do not support this hypothesis since, from their point of view, the production process is quite fast and cannot be due to the complex action of the bacteria [61]. In addition, a study performed by National Institute for Medical Research of London [13] discovered the existence of a cloud that surrounds our body. This cloud, which is possibly produced by the action of the bacteria over dead skin cells, presents four times more germs than the room air and it has a width from 1 to $4 \mathrm{~cm}$ and flows through our body at a velocity of $38 \mathrm{~m} / \mathrm{min}$.

The different types of glands are not distributed uniformly through the body, therefore the composition of our odor is different depending on the part of the body where it is captured. This difference is reported in studies with dogs [26] and in recent works analyzing the composition of the odor in the back and the arm of the same person [16]. Eccrine sweat glands are distributed all over the body, but they are specially concentrated in the palms, forehead and soles [19]. The sweat produced by these glands is quite abundant, even two or four liters per hour in a normal person doing exercise. Although, the main component of this fluid is water (up to 98\%), it also contains glicoproteins, lactic acid, sugars, amino acids and electrolytes. This sweat is originated from the extracellular fluid so it reflects the compounds present in the blood plasma [41]. In turn, apocrine glands are only present in the armpit, pubic area and areola. These are the typical sources of the bad body odor and even some authors suggest that they are also responsible of some chemical signals as pheromones [60]. Sebaceous glands are concentrated in body parts with hair such as breast, back or head. The secretions of these glands are commonly named sebum and they are rich in lipids as cholesterol, ester cholesterol, fatty acids, squalene and triglycerides. Due to the fact that the sebum is liquid at room temperature, it can flow through the whole body reaching zones without these glands like palms. The hydrolysis of these lipids produces a mixture of fatty acids which is unique for each individual [43].

Penn et al. [44] propose to capture the body odor from the sweat of the axillary region since this fluid presents a high variety of compounds. Nevertheless, other work [9] suggests that the body odor captured from the hand is much more stable since this part of the body presents a lower number of apocrine glands, whose secretions are more affected by different factors as diseases, states of mind, etc. In this work, we decided to capture the odor from the hand because its vapors are relatively easy capture as the hand is less invasive for the subject than other body parts.

This paper is organized as follows: Section 2 describes the experimental setup carried out to capture hand's odor. Section 3 identifies our odor identification system with the different stages involved in a supervised classification task, namely: pattern construction, dimensionality reduction and classification. This section also presents the methods and criteria used at each phase. Section 4 describes the evaluation methodology. Section 5 shows and analyzes the experimental results obtained by the different systems proposed in the preceding section. Finally, Section 6 states the conclusions derived from this work.

\section{Hand odor capture}

Sweat is usually collected by means of pads which have been previously sterilized in order to avoid a possible contamination of the sample. After these pads are left to rest during several hours to favor the bacteria action, the emitted vapors are captured. Nevertheless, this process needs a long time to obtain the odor profile so other works are proposing new techniques to capture the body odor in real time. In [63], the authors present a technique in which the person places the hand into a device in which air flows collecting the volatile compounds of the odor. Although this technique considerably decreases the acquisition time since it is not necessary to wait for the bacteria action, the acquisition time is about 30 min which is still high taking into account that the person must remain with his/her hand inside the device during this time.

For this work, a new sensor with a low acquisition time developed by Sociedad Europea de Análisis Diferencial de Movilidad SL (SEADM) was used. The system comprises (i) a vapor collector, which drives the skin emanations from the hand toward the ionizer, (ii) an ionization stage, where the vapors are put in contact with the cloud of ions produced by a corona discharge, and (iii) and Atmospheric Pressure Interface (API) Mass Spectrometer (MS) that collects the ions, transfers them from the atmospheric region toward the vacuum side of the MS, and finally analyzes them. The new system, which is schematically depicted in Fig. 1, resembles that described in [37], but it uses clean gas to reduce background levels, and a corona instead of the electrospray that allows the temperature of the system to be increased so as to prevent vapor deposition and the subsequent memory effects. The MS (a Qstar XL from Sciex) provides a full spectrum (number of ions detected as a function of their mass to charge ratio) every $0.1 \mathrm{~s}$. The combination of high speed and high sensitivity provided by the new system allowed the analysis of the samples to perform in real time (on-line). The raw data analyzed in this study is a series of spectrum (each being a snapshot) that describes the time evolution of the concentration of the different species. More details 


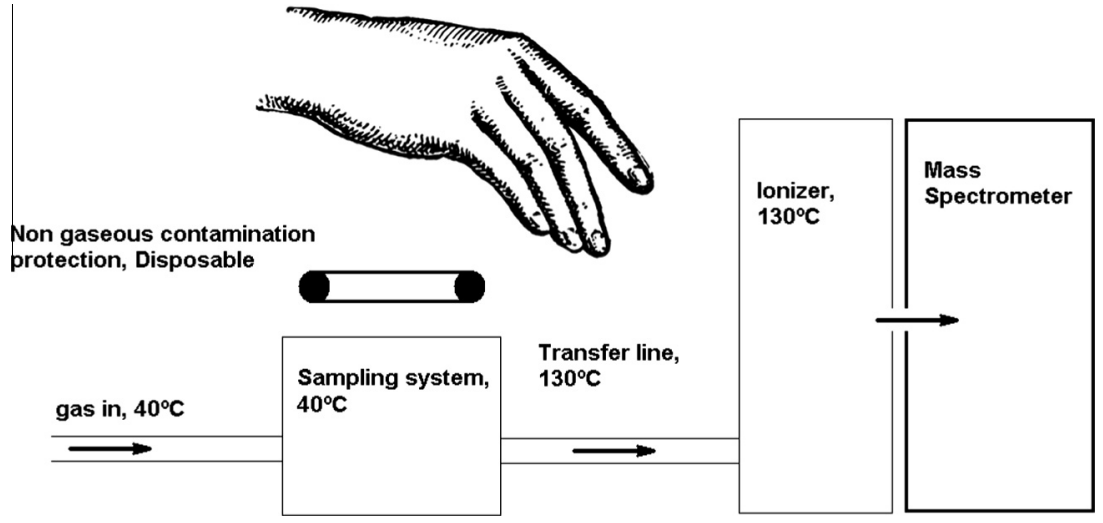

Fig. 1. Scheme of operation of the analysis system.

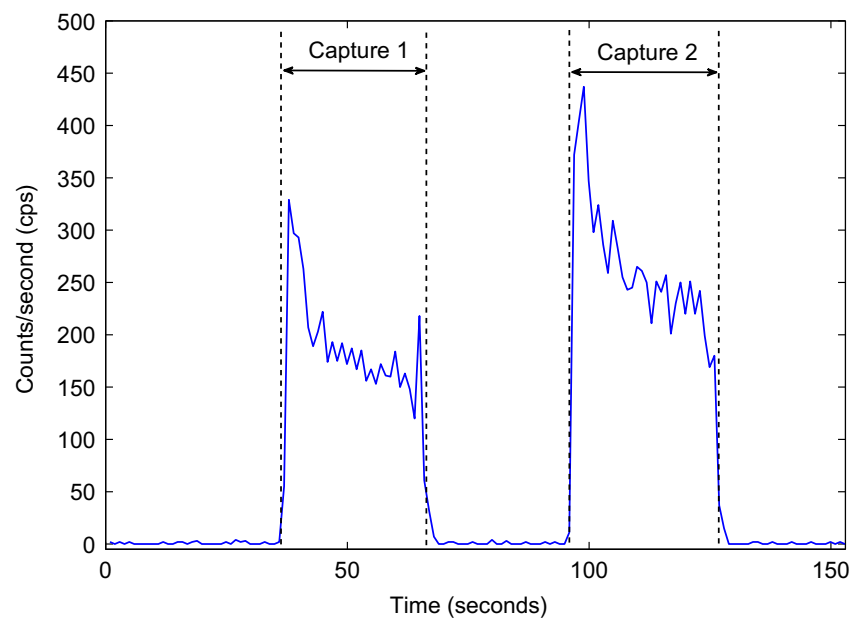

Fig. 2. Number of detected ions of lactic acid for one subject.

describing the vapor ionization systems developed by SEADM can be found in $[56,57]$.

During the campaign, the following protocol was applied: first, the subject washed and dried their hands with water, non-perfumed soap, and laboratory quality handkerchiefs (Kimwipes) in order to reduce cross contaminations effects. Then, the subject put on a sterile nitrile glove in his/her left hand cleaned with high purity methanol. The left hand was used to determine the background level of the system. To do this, the subject placed his/her left hand (wearing the cleaned glove) onto the vapor collector for $40 \mathrm{~s}$. Once the background levels were collected, the subject changed hands, now putting its nude left hand onto the vapor collector. In this step, in order to prevent non-gaseous depositions from being transferred from the skin to the vapor collector, a disposable Viton O-ring was used to separate the hand from the vapor collector. The vapors released by the right hand were then analyzed in real time during $30 \mathrm{~s}$, after which hands were switched again. In order to validate the repeatability of the measurements, the right hand was measured twice. Between the first measurement and the second measurement, the left hand was placed onto the vapor collector to provide a null input into the system so as to return to the original background level. An example of the results of these measurements is depicted in Fig. 2, where the signal of ions per second detected for a particular specie (lactic acid in this case) rises when the right hand is placed onto the vapor collector (seconds 40 and 95) and returns to the background levels when the left hand wearing a clean glove is analyzed (seconds 0,70 and 125).
The total acquisition time was longer than the time of placing the hand on the sensor because it also comprises the background measurements. However, in order to minimize the time for classification, in this work we have only used the data captured just before and during the positioning of both hands. This experiment was performed by 13 individuals ( 8 males and 5 females) in 28 different sessions conducted in different days to ensure the permanence of the analyzed features. Although in some works the participants of the experiment must wear special clothes [10] or perform physical activity to increase their production of sweat $[9,63]$, in this work the participants were not asked to do any previous exercise or wear any special clothes.

\section{Identification system}

Once the samples are collected, the problem of odor identification can be tackled from the point of view of machine learning $[36,20]$. In particular, the problem of subject identification from their body odor can be identified with a supervised multi-class classification task [4] in which each class corresponds to a subject and the classification algorithm (also known as classifier or learning algorithm) must find the dependences between the input data and the classes. After training the system with several samples of each individual, the classification model can be used to determine the discriminatory information to be used to predict the class of unlabeled samples. The performance of the system is calculated by means of the recognition rate (or classification accuracy), which indicates the percentage of the testing samples correctly classified that is, the number of subjects correctly identified. This is not the first attempt to tackle mass-spectrometry data from the point of view of machine learning and several works are published in this line as pointed out in the review of Saeys et al. [49].

Any supervised classification system is composed of different stages as the ones shown in Fig. 3. The data acquisition phase corresponds to the process described in Section 2 that gave as result the hand odor of both hands from 13 subjects captured in 28 sessions carried out in different days. The adaptation of the remaining

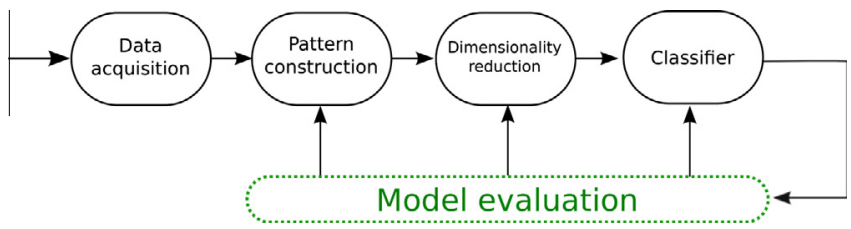

Fig. 3. Diagram of the design of a machine learning system. Figure adapted from [14]. 
stages to our identification system will be presented in the following subsections.

\subsection{Pattern construction}

Once captured, the experimental samples should be expressed in the correct format to be interpreted by the classifier. More precisely, the information related to each session will be a pattern and each pattern will be defined as a set of variables (attributes or features) created according to certain criteria. This way, a pattern can be understood as a multidimensional vector, being each dimension one of these variables. In our case, the acquisition system provides a mass spectrogram for each session. This spectrogram reflects the number of ions detected for each mass during the time each subject was placing the hand in the sensor and every compound can be identified with a peak in the spectrogram. Therefore, our attributes will be the different mass intervals corresponding to each peak which provides information about a compound of the body odor. Initially, only those mass intervals corresponding to fatty acids were identified based on our previous work [37]; however, these intervals showed to be insufficient to classify people accurately. Peak detection was refined by performing a visual inspection of all the spectrograms, giving as a result 752 common intervals for all the subjects. This means that each sample of body odor captured by the acquisition system will be represented by a vector of 752 dimensions, each of which indicates the number of ions (intensity) that have fallen into the corresponding interval.

Having defined the configuration of the patterns, a numerical analysis revealed that the acquisition system accumulated noise coming from the previous captures. Thanks to the background signal measured just before and during both hands were placed, the contribution of this noise was subtracted in order to obtain the pure signal independent of previous samples and thus, characteristic of each individual. Furthermore, due to the fact that the concentration of the ions may depend on different environmental factors, such as humidity, temperature or even the hour of capture, these measurements were normalized. This normalization consisted in dividing the measurement for each interval by the total number of ions detected by the acquisition system along all the intervals in the same session. Consequently, the entries of the vector corresponding to each body odor sample represent the relative concentration of each identified compound.

\subsection{Dimensionality reduction}

The characteristics of the identification system described in the preceding sections are in line with those commonly appearing in bioinformatics problems: large dimensionality and small sample sizes. This kind of problems is known as small sample size problems and much effort has been invested in bioinformatics, machine learning and data mining areas to develop dimensionality reduction algorithms capable of dealing with the inherent risk of imprecision and overfitting [49]. Specifically, as described in Sections 2 and 3.1, in our identification problem the number of features (752 mass intervals) is much larger than the number of samples per class (56 samples per subject).

Two different approaches were evaluated for this identification problem: feature selection and feature extraction. Feature selection techniques select the most relevant subset of features from the original set of variables [22]. Among feature selection approaches, three groups can be found as a function of their dependence on the classifier: (i) filter methods are independent of the classifier and they are based on intrinsic characteristics of the data; (ii) wrapper methods use the classifier as a black-box to evaluate the goodness of a candidate subset of features, and (iii) embedded methods are completely linked to the classifier as they incorporate the feature selection task in the objective function of the classifier. Additionally, feature selection algorithms can be divided into feature subset selection (FSS) methods and feature ranking approaches. FSS algorithms give as a result a closed subset of features while feature ranking approaches provide a list of all the original features sorted according to certain criterion of relevance.

According to the comparative study carried out by Saeys et al. [49], filters are the most common algorithms in mass-spectrometry domains. In particular, univariate filter methods, which only consider dependences between the attributes and the class, are the most used approach. Though multivariate filters extend the functionality of the univariate approach by taking also into account interactions among features, they are not so widespread in the mass-spectrometry domain. Wrapper algorithms have been successfully applied in several influential works. Finally, embedded approaches are emerging as a promising alternative. For more information and a detailed review of feature selection in massspectrometry domains, the reader is referred to Ref. [49].

Regarding feature extraction methods, they apply a transformation that projects the data into a lower dimensional space. These approaches have also been successfully used in mass-spectrometry data $[58,33,38,46,1,34,39]$. In the following sections, the application of feature selection and feature extraction algorithms to our problem is described in more detail.

\subsubsection{Feature selection}

As pointed out in Section 3.2, feature selection algorithms can be divided into different groups according to the dependence between the selector and the classifier. In this work, filter and wrapper approaches have been considered since the first one is the most commonly used in mass-spectrometry domains [49] and the second one generally achieves good recognition rates [22,49]. Embedded algorithms were not taken into account as they do not provide flexibility to be easily tested with different classifiers.

Filter methods are independent of the classifier since they are based on intrinsic properties of the data such as correlations, mutual information or distances. These approaches can be divided into two subgroups: (i) univariate filters, which only consider dependences between the attributes and the class label, and (ii) multivariate filters that also model dependences among features. The former group is computationally faster but it usually yields poorer results, especially if there is high redundancy in the data [22]. In this work, we considered a multivariate filter approach as we expected our features to be highly redundant due to the overlapping of mass intervals and the dependences among different compounds, even when their mass intervals do not overlap. In particular, we opted for ReliefF [29], a feature ranking algorithm that evaluates the quality of a feature according to how well it distinguishes between instances that are near to each other. Specifically, the algorithm starts by identifying the closest samples of the same class (nearest hits) and the closest samples from different classes (nearest misses) of each training pattern and those features that keep close samples in the same class while getting away samples from different classes are preferred. Therefore, ReliefF is an appealing solution to our problem since it selects the characteristic compounds of each user, rewarding those compounds that take similar values among the samples of the user but different values in the samples belonging to other individuals. Additionally, ReliefF was chosen because (i) it has already been used in mass-spectrometry domains [45]; (ii) it is a multivariate filter feature selection algorithm which does not require many training samples to obtain reliable estimations of the feature scores; (iii) it can be easily formulated for multiclass classification problems; and (iv) it can be applied before any classification algorithm.

Wrapper methods use the classifier performance to evaluate the goodness of a subset of features. However, considering all the 
possible subsets of features is an unapproachable task and different approximations are suggested in the literature to address this problem [22]. In this work, sequential forward feature selection technique was considered as it is one of the most commonly used algorithms. This is a greedy technique in which the search of the best subset is performed by adding iteratively the most relevant features to an initially empty subset. At each iteration, the most relevant feature is that producing the highest recognition rate and in case of tie, one of the candidates is randomly chosen. Due to this randomly selection, the algorithm should be restarted several times to explore different combinations of features. The inclusion of new features stops when either the maximum recognition rate is achieved (100\%) or the classification rate converges. Thus, the sequential forward feature selection procedure can be viewed as a feature subset selection approach if the algorithm stops before all the original features are added to the final subset of attributes, but it also can be formulated as a ranking feature selection method by considering the order in which the features have been incorporated to the final subset of variables.

\subsubsection{Feature extraction}

Among feature extraction techniques, Principal Component Analysis (PCA) [14,27] was tested because it is particularly interesting for our identification system as it allows ignoring those compounds with a low variance and therefore, not helpful to discriminate between different subjects. Furthermore, PCA has shown good performance in a wide range of domains [54,64,47], including mass-spectrometry problems $[58,33,38,1,34,39]$. Although PCA is an unsupervised technique, it can efficiently complement a supervised classifier in order to achieve the objective of the system. In fact, any classifier can be used in conjunction with PCA since it does not make any kind of assumption about the subsequent classification model.

The idea behind PCA is to find a set of orthogonal directions to project the data into a lower-dimensional space while preserving as much of the variance as possible. PCA does not take into account the label of the data (in our case the owner of the body odor) but it attempts to find a set of orthogonal directions that maximize the data variance. The result of PCA is a list of orthonormal directions ordered according to the percentage of explained variance, i.e., the first component is that explaining the highest proportion of the data variance, and so on. Thus, the dimension of the problem is reduced by selecting the first principal components and projecting the data into the subspace defined by these directions. As PCA gives as a result an ordered sequence of directions to project the data, it can viewed as ranking feature extraction approach.

\subsection{Classifier}

In this work, different classifiers were considered to discriminate the body odor of each subject. It is fundamental to note that, given the characteristics of the data we are working with, simple models are preferred to avoid the overfitting phenomenon $[55,40]$. Specifically, we have tested five types of classifiers, namely k Nearest Neighbors (k-NN), Linear Discriminant Analysis (LDA), Logistic Regression, Naive Bayes and linear Support Vector Machines (SVMs) [14,4]. Though different approaches motivated these algorithms, all of them are simple, easily adaptable to the multiclass scenario and they have already been used in the electronic nose domain $[21,5,59,18,15]$.

Note that LDA is commonly used for dimensionality reduction before later classification but in our case, we have used it as a linear classifier since LDA formulation is ill-posed when applied to our entire dataset and thus, it must be preceded by a dimensionality reduction step. When used as classifier, LDA projects samples into the subspace defined by LDA's directions and then assigns to each pattern the class with the closest projected mean.

Nonlinear SVMs (RBF kernel) were also included in the further experiments to give evidence of the inadequacy of more complex models for our identification system.

Finally, observe that these classifiers play a different role depending on the dimensionality reduction method to apply. While in wrapper algorithms the classification and feature selection processes are completely linked, filters and PCA are completely independent of the classifier to be used.

\section{Experimental setup}

It is fundamental to define a rigorous evaluation method to determine whether the requirements of the identification system are fulfilled or not. A common practice to evaluate a machine learning system consists in dividing the data into three disjoint subsets: (i) a training set to learn the parameters of the classification model, (ii) a validation set to tune meta-parameters of the system - in our case, those associated with the classifier and the number of features to retain, and (iii) a test set formed by unseen data that have not been used to adjust any parameters or metaparameters and which allows us to reliably evaluate the performance of the system when deployed in an operating environment. Ideally, the classification system has to be specific enough to infer the relation between the patterns and the classes, but general enough to correctly predict the label of those patterns not seen during the training phase.

Although there are different techniques to divide the total dataset into these three subsets, in this case we adopted a variation of the leave-one-out (LOO) strategy [4]. Taking into account that the number of samples per subject ( 2 captures $\times 28$ sessions) is not too large, the LOO approach is appropriate since it maximizes the number of samples to be used during the training phase and each pattern is used once as validation data. Specifically, given the entire dataset, the LOO method keeps a single sample as the validation data and the remaining samples are used to train the model. This process is repeated as many times as the number of samples in the data by leaving aside one of the patterns at each time.

The methodology here implemented can be viewed as a LOO strategy at session level which is used in both validation and testing phases as shown in Fig. 4. First of all, it should be noted that the data coming from the same session (two captures per session) were preserved in the same training/validation/test partition in order to avoid artificially high recognition rates due to the temporal dependence of both measurements. At the first level, one session per subject was kept for the testing set. Ideally, all the possible combinations coming from leaving one sample per subject should be considered. However, this approach is computationally intensive. Thus, each testing set was configured by taking the first session for all the subjects, the second session for all the subjects, etc. This way, 28 different test sets were obtained. The same strategy was applied over the remaining 27 sessions (per subject): the $n$th session of each subject was kept for validation while the remaining 26 sessions were used to train the model and this process was repeated 27 times. Note that strictly speaking we did not apply LOO since both validation and test sets comprised 26 samples instead of the single pattern characteristic of the LOO approach.

In particular, the training set was used to select/extract the most relevant features and to train the classifiers. The validation set was used to adjust the best number of features in each case as well as the classifiers' meta-parameters. It also guided the feature search in the wrapper approach. The final performance of the identification system was measured over the test set in order 


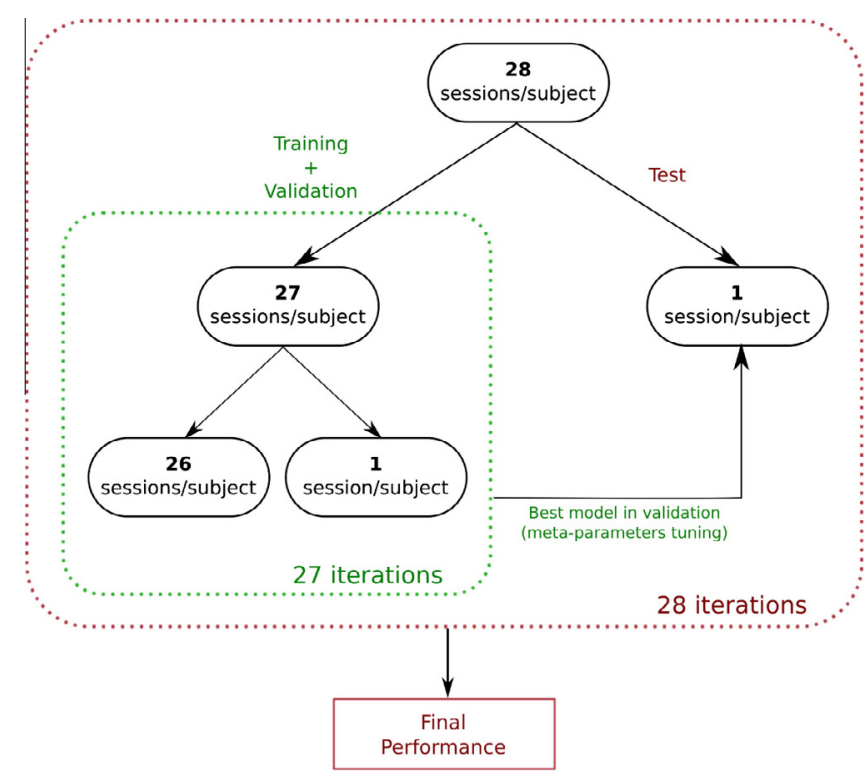

Fig. 4. Experimental methodology used to generate the training, validation and test partitions.

to obtain a reliable estimation of its effectiveness once working in a real environment.

According to Fig. 1 and the ideas presented in Sections 3.2 and 3.3, different combinations of dimensionality reduction techniques and classifiers were tested in order to evaluate the feasibility of using the body odor as biometric identifier. The algorithms considered in our analysis are listed in Table 1.

All the dimensionality reduction methods (ReliefF, PCA and the wrapper approach) are feature ranking approaches that require specifying the dimension of the reduced space which, in turn, will be the input space for the subsequent classifier. ReliefF also requires specifying the number of nearest neighbors from the same class (nearest hits) and from different classes (nearest misses) to consider in the computation of each feature score. The results here shown are those corresponding to a neighborhood of size 3. Neighborhoods of size 5 and 7 were also implemented with no better results. Finally, some meta-parameters need also to be adjusted for some of the classification methods in Table 1. The grid of values considered for each meta-parameter is shown in Table 2. Note that the maximum number of features selected with the wrapper algorithm is 150 while it is 250 for ReliefF and PCA. This fact is due to the high computational cost of the wrapper algorithm [49] and its poor generalization performance in our data, as it will be shown in Section 5. Then, according to the experimental setup above described, the optimal value of these parameters in each case is that minimizing the classification error in the validation set.

Table 1

Dimensionality reduction techniques and classifiers considered in our experimental setup.

\begin{tabular}{llll}
\hline Dimensionality reduction & & Classifier \\
\hline Feature selection & Feature extraction & \\
\hline ReliefF & PCA & k-NN \\
& & LDA \\
& & Logistic Regression \\
& & Naive Bayes \\
& & Linear SVM \\
& RBF SVM \\
Wrapper (Forward) & - & LDA \\
\hline
\end{tabular}

Table 2

Meta-parameter grids considered in our experiments.

\begin{tabular}{lll}
\hline Algorithm & $\begin{array}{l}\text { Meta- } \\
\text { parameter }\end{array}$ & Grid \\
\hline ReliefF & \# Features & $\{1,2, \ldots, 9,10,20,30, \ldots, 200,250\}$ \\
PCA & \# Features & $\{1,2, \ldots, 9,10,20,30, \ldots, 200,250\}$ \\
Wrapper & \# Features & $\{1,2, \ldots, 9,10,20,30, \ldots, 150\}$ \\
k-NN & k & $\{1,3,5,7,9\}$ \\
Linear SVM & Cost parameter & $\left\{10^{-4}, 10^{-3}, 10^{-2}, 10^{-1}, 1,10^{1}, 10^{2}, 10^{3}, 10^{4}\right\}$ \\
RBF SVM & Cost parameter & $\left\{10^{-4}, 10^{-3}, 10^{-2}, 10^{-1}, 1,10^{1}, 10^{2}, 10^{3}, 10^{4}\right\}$ \\
RBF SVM & Kernel width & $\left\{10^{-3}, 10^{-2}, 10^{-1}, 1,10^{1}, 10^{2}, 10^{3}\right\}$ \\
\hline
\end{tabular}

\section{Results and discussion}

As point of reference, note that the average recognition rate of a random classifier for this problem is $7.69 \%$ since there are 13 subjects (classes) and all of them have the same number of samples. This rate can be used as a reference in order to verify the presence of discriminatory information in the hands' odor.

First of all, we will analyze the results obtained for the wrapper algorithm to show its poor performance in our case. The wrapper algorithm was implemented as a sequential forward feature selection technique using LDA as classifier. Fig. 5 shows the average recognition rates for the validation and test partitions as a function of the number of features. It can be observed that the results for the test partition are significantly worse than those obtained with the validation set. Overfitting is a well-known limitation of wrapper approaches $[22,49]$ : the feature selection process is guided by the performance of the classifier in the validation data and therefore, it is likely that the obtained model overfits the validation samples and it does not generalize well to unseen data. In spite of this decrease with respect to the validation results, the average recognition rate for the testing data using only 30 features is about $74.75 \%$ ( $81 \%$ in validation) which implies an improvement of nine times over the random classifier (7.69\%). Furthermore, when 95 features are considered, the recognition rate over the testing set reaches its maximum at $77.75 \%$ ( $85.73 \%$ in validation), which improves in 10 times the effectiveness of the random classifier. Although these results are promising, the wrapper solution was dismissed in favor of the other dimensionality reduction techniques that yield better performance and generalization in the test set besides lower execution times.

Tables 3 and 4 show the classification accuracy (\%) for ReliefF and PCA as a function of the number of features and principal components, respectively, and when combined with the classifiers listed in Table 1. The effectiveness of the classifiers when dimensionality reduction is not applied is also shown as reference except for LDA that is ill-posed when all the original features are taken into account (the number of features is larger than the number of samples). When applicable, the results are those corresponding to the best meta-parameters obtained from the validation set. In order to provide a visual representation of the results that makes it easier to compare them, Figs. 6 and 7 show the test classification rates (\%) of ReliefF and PCA, respectively, as well as the best performance obtained with no dimensionality reduction and corresponding to the linear SVM (see Tables 3 and 4). Note that, according to the experimental protocol described in Section 4, the training sets are similar to each other and thus, the estimate of error is almost unbiased but it can have high variance [6] and [23, Chapter 7].

First of all, note that in both cases the dimensionality reduction phase not only reduces the risk of overfitting and the computational cost of the classifier but it also improves the effectiveness of the identification system by removing noisy and irrelevant features. Furthermore, generally speaking, the results obtained when 


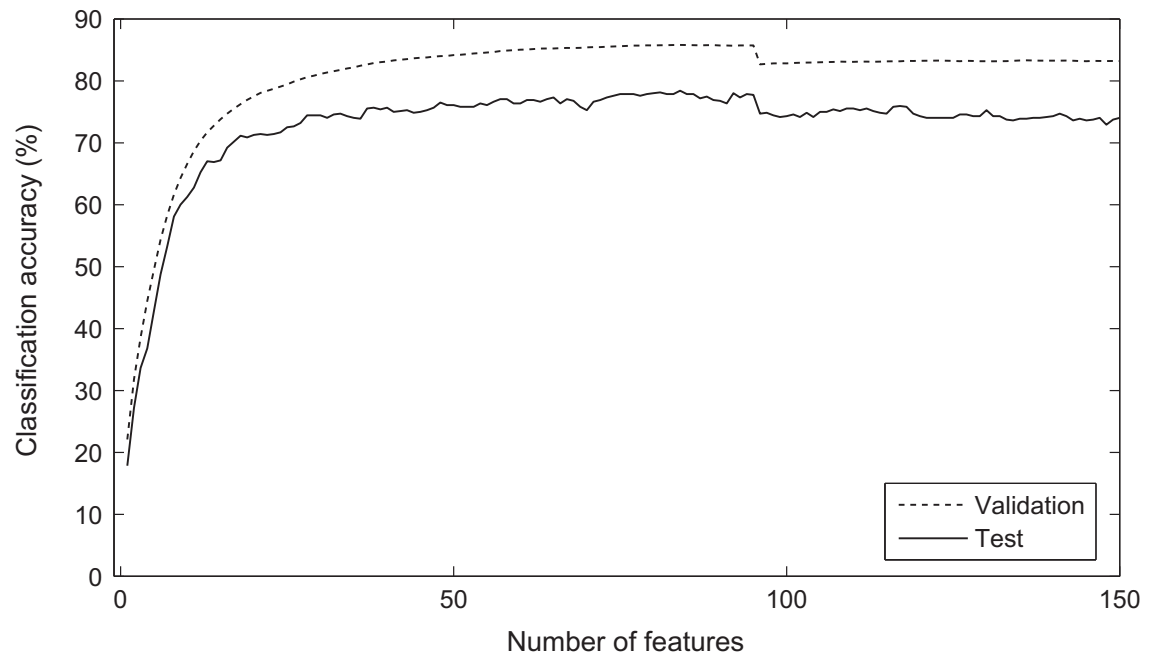

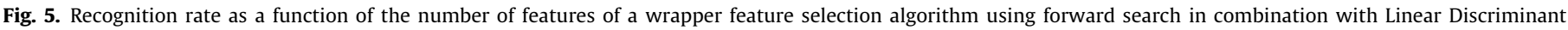
Analysis. Results over the validation and test partitions are shown.

Table 3

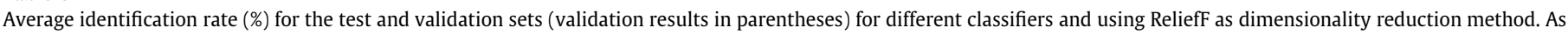

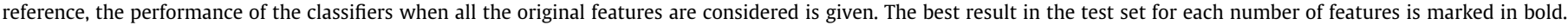

\begin{tabular}{|c|c|c|c|c|c|c|c|}
\hline \multirow[t]{2}{*}{ Class. } & \multicolumn{6}{|c|}{ Number of features } & \multirow[t]{2}{*}{ No FS } \\
\hline & 1 & 10 & 50 & 100 & 200 & 250 & \\
\hline$k-N N$ & $\begin{array}{c}18.68 \pm 1.41 \\
(19.57 \pm 0.74)\end{array}$ & $\begin{array}{c}40.25 \pm 1.53 \\
(43.15 \pm 0.85)\end{array}$ & $\begin{array}{c}53.02 \pm 2.33 \\
(53.91 \pm 0.72)\end{array}$ & $\begin{array}{c}55.08 \pm 2.67 \\
(54.50 \pm 2.22)\end{array}$ & $\begin{array}{c}50.55 \pm 2.24 \\
(50.71 \pm 0.82)\end{array}$ & $\begin{array}{c}49.18 \pm 2.21 \\
(50.80 \pm 0.80)\end{array}$ & $\begin{array}{c}39.15 \pm 2.03 \\
(40.48 \pm 0.67)\end{array}$ \\
\hline$L D A$ & $\begin{array}{c}19.37 \pm 1.06 \\
(19.46 \pm 0.40)\end{array}$ & $\begin{array}{c}44.37 \pm 2.38 \\
(44.93 \pm 0.87)\end{array}$ & $\begin{array}{c}73.90 \pm 2.46 \\
(72.99 \pm 1.11)\end{array}$ & $\begin{array}{c}76.65 \pm 2.43 \\
(76.97 \pm 1.21)\end{array}$ & $\begin{array}{c}76.92 \pm 2.37 \\
(76.71 \pm 1.03)\end{array}$ & $\begin{array}{c}76.37 \pm 2.02 \\
(75.85 \pm 0.86)\end{array}$ & - \\
\hline LogReg & $\begin{array}{c}\mathbf{1 9 . 5 1} \pm \mathbf{0 . 9 5} \\
(19.73 \pm 0.60)\end{array}$ & $\begin{array}{c}\mathbf{5 5 . 9 1} \pm \mathbf{2 . 4 2} \\
(55.97 \pm 0.94)\end{array}$ & $\begin{array}{c}71.29 \pm 2.16 \\
(69.11 \pm 1.41)\end{array}$ & $\begin{array}{c}71.29 \pm 2.18 \\
(71.15 \pm 2.15)\end{array}$ & $\begin{array}{c}71.84 \pm 2.19 \\
(71.37 \pm 1.58)\end{array}$ & $\begin{array}{c}71.15 \pm 2.47 \\
(70.98 \pm 1.23)\end{array}$ & $\begin{array}{c}13.19 \pm 0.96 \\
(11.99 \pm 1.20)\end{array}$ \\
\hline $\begin{array}{l}\text { Naive } \\
\text { Bayes }\end{array}$ & $\begin{array}{c}19.37 \pm 1.06 \\
(19.46 \pm 0.40)\end{array}$ & $\begin{array}{c}41.21 \pm 1.96 \\
(41.29 \pm 1.04)\end{array}$ & $\begin{array}{c}54.40 \pm 1.92 \\
(53.85 \pm 0.96)\end{array}$ & $\begin{array}{c}55.22 \pm 1.91 \\
(55.39 \pm 1.15)\end{array}$ & $\begin{array}{c}53.98 \pm 2.14 \\
(54.36 \pm 1.04)\end{array}$ & $\begin{array}{c}53.02 \pm 2.33 \\
(52.84 \pm 0.80)\end{array}$ & $\begin{array}{c}31.45 \pm 1.76 \\
(31.36 \pm 0.72)\end{array}$ \\
\hline $\begin{array}{l}\text { Linear } \\
\text { SVM }\end{array}$ & $\begin{array}{c}7.69 \pm 0.00 \\
(7.73 \pm 0.07)\end{array}$ & $\begin{array}{c}45.74 \pm 1.43 \\
(45.80 \pm 1.10)\end{array}$ & $\begin{array}{c}\mathbf{8 1 . 7 3} \pm \mathbf{1 . 9 6} \\
(80.32 \pm 1.15)\end{array}$ & $\begin{array}{c}\mathbf{8 3 . 1 0} \pm \mathbf{2 . 0 0} \\
(82.96 \pm 1.28)\end{array}$ & $\begin{array}{c}\mathbf{8 3 . 9 3} \pm \mathbf{2 . 2 4} \\
(83.66 \pm 0.76)\end{array}$ & $\begin{array}{c}\mathbf{8 4 . 0 7} \pm \mathbf{2 . 0 0} \\
(83.24 \pm 0.92)\end{array}$ & $\begin{array}{c}\mathbf{7 2 . 8 0} \pm \mathbf{2 . 6 5} \\
(72.96 \pm 1.25)\end{array}$ \\
\hline $\begin{array}{l}R B F \\
S V M\end{array}$ & $\begin{array}{c}17.17 \pm 1.11 \\
(19.75 \pm 0.82)\end{array}$ & $\begin{array}{c}28.16 \pm 1.81 \\
(28.28 \pm 0.90)\end{array}$ & $\begin{array}{c}29.12 \pm 1.62 \\
(28.94 \pm 1.05)\end{array}$ & $\begin{array}{c}32.55 \pm 2.23 \\
(30.48 \pm 3.77)\end{array}$ & $\begin{array}{c}28.71 \pm 1.66 \\
(29.33 \pm 2.36)\end{array}$ & $\begin{array}{c}35.85 \pm 2.08 \\
(35.78 \pm 1.69)\end{array}$ & $\begin{array}{c}29.26 \pm 1.69 \\
(29.12 \pm 0.55)\end{array}$ \\
\hline
\end{tabular}

Table 4

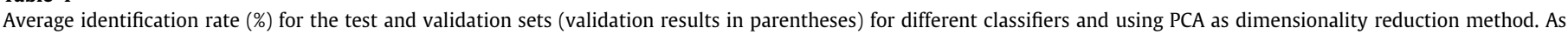

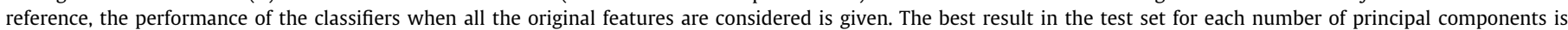
marked in bold.

\begin{tabular}{|c|c|c|c|c|c|c|c|}
\hline \multirow[t]{2}{*}{ Class. } & \multicolumn{6}{|c|}{ Number of principal components } & \multirow[t]{2}{*}{ No FS } \\
\hline & 1 & 10 & 50 & 100 & 200 & 250 & \\
\hline$k-N N$ & $\begin{array}{c}13.32 \pm 1.07 \\
(14.41 \pm 0.98)\end{array}$ & $\begin{array}{c}38.74 \pm 1.80 \\
(39.27 \pm 0.62)\end{array}$ & $\begin{array}{c}39.56 \pm 2.07 \\
(40.43 \pm 0.70)\end{array}$ & $\begin{array}{c}39.29 \pm 2.12 \\
(40.57 \pm 0.68)\end{array}$ & $\begin{array}{c}39.15 \pm 2.01 \\
(40.52 \pm 0.68)\end{array}$ & $\begin{array}{c}39.42 \pm 1.94 \\
(40.54 \pm 0.70)\end{array}$ & $\begin{array}{c}39.15 \pm 2.03 \\
(40.48 \pm 0.67)\end{array}$ \\
\hline$L D A$ & $\begin{array}{c}\mathbf{1 6 . 2 1} \pm \mathbf{1 . 3 0} \\
(16.20 \pm 0.70)\end{array}$ & $\begin{array}{c}39.70 \pm 2.43 \\
(39.25 \pm 0.62)\end{array}$ & $\begin{array}{r}64.84 \pm 3.30 \\
(64.84 \pm 0.79)\end{array}$ & $\begin{array}{c}\mathbf{7 8 . 1 6} \pm \mathbf{2 . 4 9} \\
(78.00 \pm 0.74)\end{array}$ & $\begin{array}{c}\mathbf{8 2 . 5 5} \pm \mathbf{1 . 8 6} \\
(82.28 \pm 0.61)\end{array}$ & $\begin{array}{c}\mathbf{8 1 . 7 3} \pm \mathbf{2 . 0 5} \\
(81.36 \pm 0.75)\end{array}$ & - \\
\hline LogReg & $\begin{array}{c}14.56 \pm 1.11 \\
(14.78 \pm 0.82)\end{array}$ & $\begin{array}{c}\mathbf{4 7 . 8 0} \pm \mathbf{2 . 4 2} \\
(47.90 \pm 0.89)\end{array}$ & $\begin{array}{c}\mathbf{6 6 . 4 8} \pm \mathbf{2 . 0 5} \\
(64.22 \pm 0.94)\end{array}$ & $\begin{array}{c}75.00 \pm 2.09 \\
(75.02 \pm 0.96)\end{array}$ & $\begin{array}{c}79.12 \pm 1.98 \\
(79.70 \pm 0.79)\end{array}$ & $\begin{array}{c}79.53 \pm 2.08 \\
(79.23 \pm 0.92)\end{array}$ & $\begin{array}{c}13.19 \pm 0.96 \\
(11.99 \pm 1.20)\end{array}$ \\
\hline $\begin{array}{l}\text { Naive } \\
\text { Bayes }\end{array}$ & $\begin{array}{c}\mathbf{1 6 . 2 1} \pm \mathbf{1 . 3 0} \\
(16.20 \pm 0.70)\end{array}$ & $\begin{array}{c}38.87 \pm 2.30 \\
(38.28 \pm 0.89)\end{array}$ & $\begin{array}{c}61.68 \pm 3.11 \\
(61.16 \pm 0.86)\end{array}$ & $\begin{array}{c}76.65 \pm 2.63 \\
(76.39 \pm 0.56)\end{array}$ & $\begin{array}{c}82.14 \pm 2.19 \\
(81.33 \pm 0.72)\end{array}$ & $\begin{array}{l}80.91 \pm 2.25 \\
(80.89 \pm 0.77)\end{array}$ & $\begin{array}{c}31.45 \pm 1.76 \\
(31.36 \pm 0.72)\end{array}$ \\
\hline $\begin{array}{l}\text { Linear } \\
\text { SVM }\end{array}$ & $\begin{array}{c}13.19 \pm 0.54 \\
(13.55 \pm 0.33)\end{array}$ & $\begin{array}{c}31.87 \pm 1.42 \\
(32.95 \pm 0.86)\end{array}$ & $\begin{array}{c}53.71 \pm 2.26 \\
(53.92 \pm 1.15)\end{array}$ & $\begin{array}{c}63.19 \pm 2.49 \\
(63.50 \pm 1.05)\end{array}$ & $\begin{array}{c}66.48 \pm 2.68 \\
(66.60 \pm 0.87)\end{array}$ & $\begin{array}{c}65.52 \pm 1.91 \\
(67.35 \pm 1.32)\end{array}$ & $\begin{array}{r}\mathbf{7 2 . 8 0} \pm \mathbf{2 . 6 5} \\
(72.96 \pm 1.25)\end{array}$ \\
\hline $\begin{array}{l}R B F \\
S V M\end{array}$ & $\begin{array}{c}12.50 \pm 0.92 \\
(13.14 \pm 1.13)\end{array}$ & $\begin{array}{c}8.93 \pm 0.53 \\
(8.92 \pm 0.18)\end{array}$ & $\begin{array}{c}7.97 \pm 0.19 \\
(7.95 \pm 0.08)\end{array}$ & $\begin{array}{c}7.97 \pm 0.19 \\
(7.96 \pm 0.07)\end{array}$ & $\begin{array}{c}7.97 \pm 0.19 \\
(7.96 \pm 0.07)\end{array}$ & $\begin{array}{c}7.97 \pm 0.19 \\
(7.96 \pm 0.07)\end{array}$ & $\begin{array}{c}29.26 \pm 1.69 \\
(29.12 \pm 0.55)\end{array}$ \\
\hline
\end{tabular}

used ReliefF and PCA reveal that these two approaches better face our identification problem than the wrapper algorithm. The superiority of ReliefF and PCA is not surprising. We are facing a smallsample-size problem with high risk of overfitting and algorithms like ReliefF and PCA, based on intrinsic properties of the data, are able to better generalize given that the validation set is only used to decide the optimum number of variables but not to select them as in the case of wrapper approaches. 


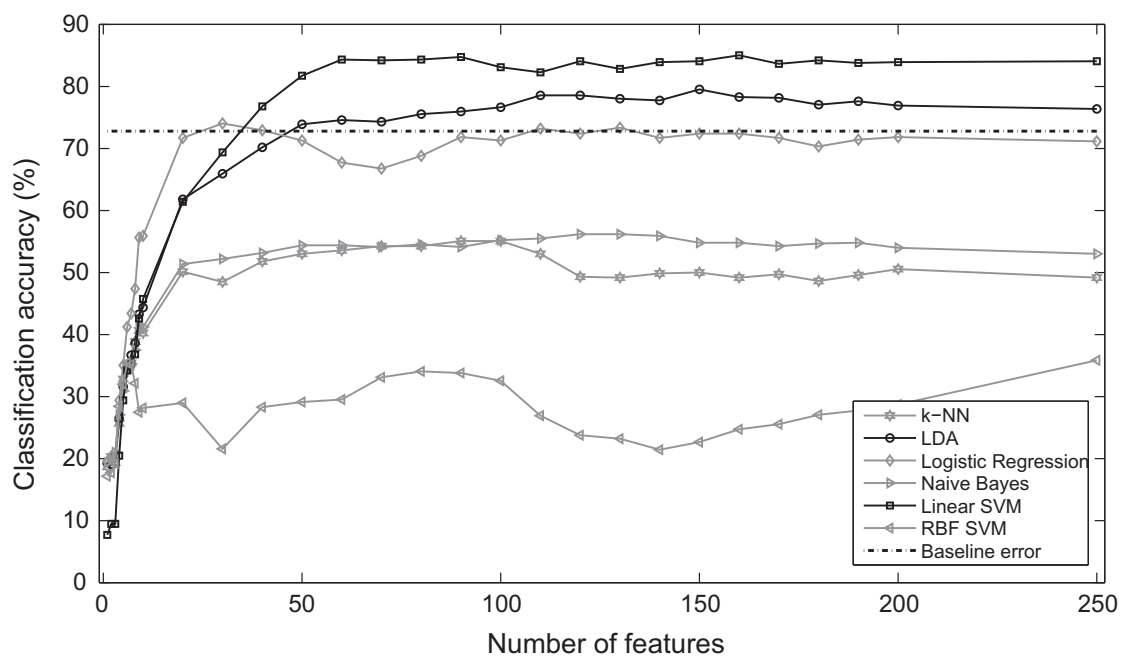

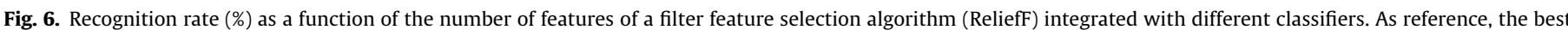
performance obtained by considering all the original features is also shown (Baseline error). Results over the test partition are shown.

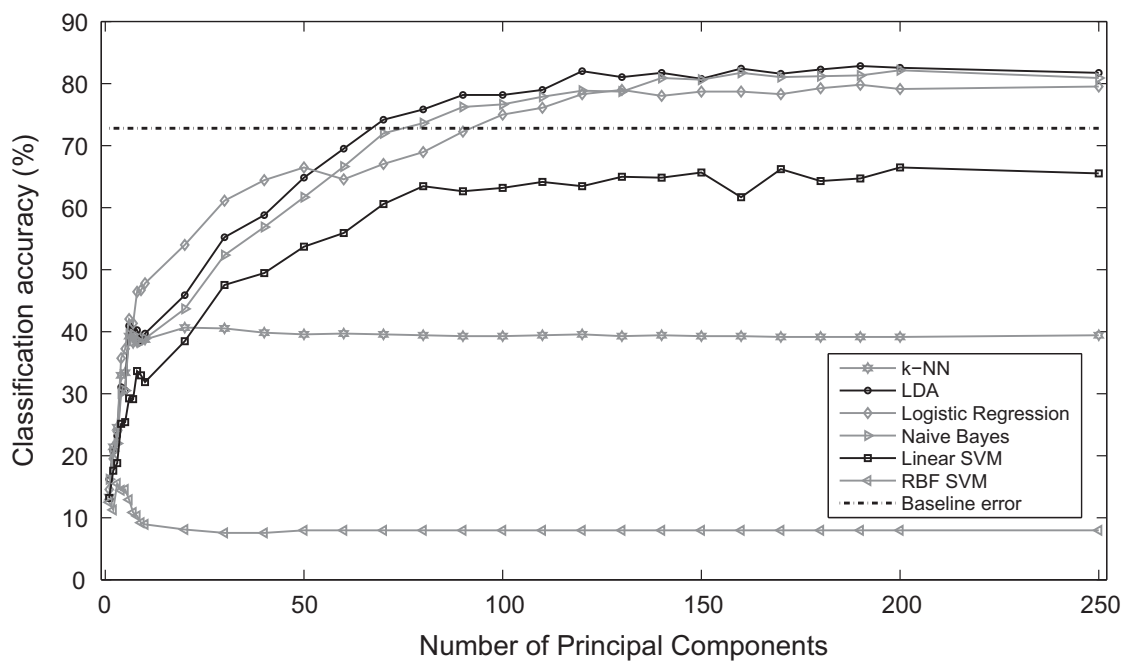

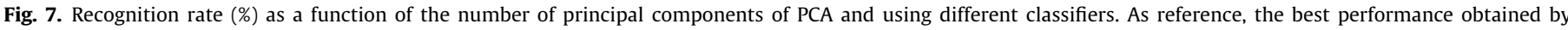
considering all the original features is also shown (Baseline error). Results over the test partition are shown.

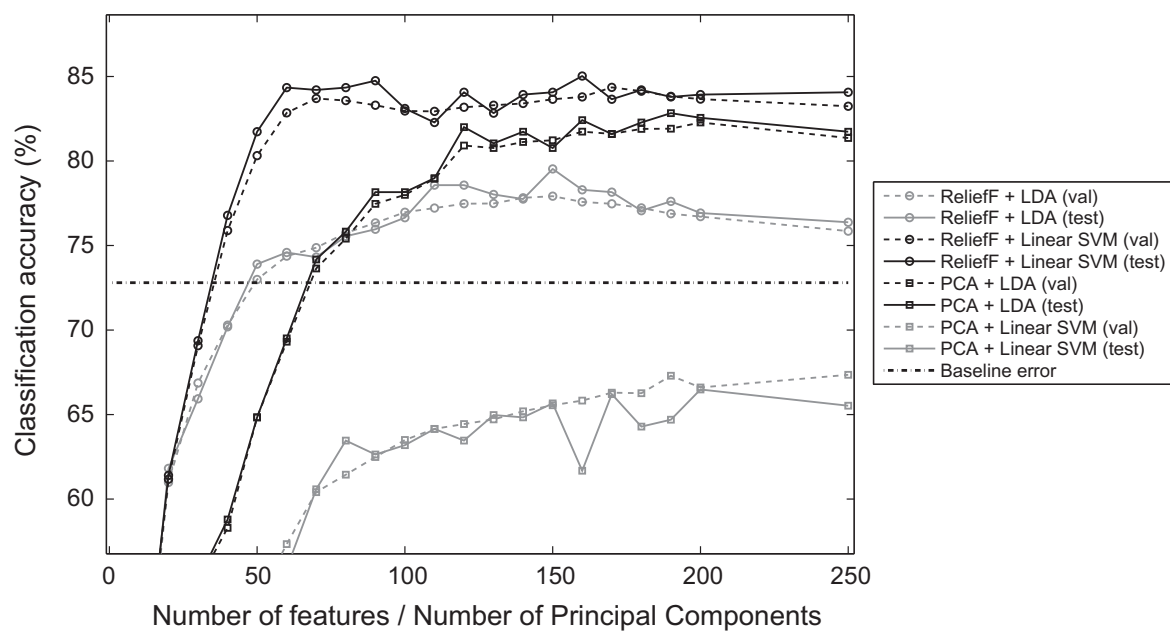

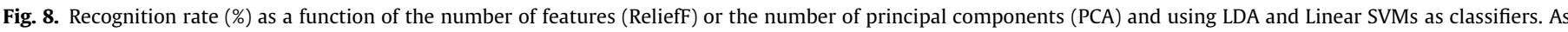

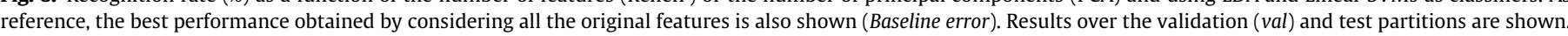


Fig. 6 shows the good performance of the system when combining ReliefF and a linear SVM, achieving recognition rates of $85.03 \%$ in the test set when 160 features are taking into account, which implies an improvement of more than 11 times with respect to the random classifier and a reduction of the dimensionality of the problem by a factor of 4.7 (752 original features/160 selected features). Actually, ReliefF has been already successfully used in mass-spectrometry domains [45]. Regarding PCA (Fig. 7), the highest recognition rate for the test data is achieved in combination with LDA when 190 principal components are kept. The accuracy amounts to $82.83 \%$, which means that PCA + LDA outperforms the random classifier in 10.77 times while reducing the dimension of the problem by a factor of 3.96 ( 752 original features/190 principal components). This good performance is also corroborated by the successful combination of PCA and LDA in previous works addressing mass-spectrometry problems $[38,33,1,34]$. Finally, remark that it is not strange that both linear SVMs and LDA behave similarly in some cases given the close relationship between LDA and the Fisher Discriminant Analysis (FDA) and the equivalence between FDA and SVMs [48].

Regarding the other classifiers, k-NN does not yield good results in either case, possibly because it requires scale of the features to be consistent with the importance of the variable. This is not the case here: there are compounds that are abundant and common to all people (such as lactic acid) and they are not necessarily discriminative. Logistic Regression and Naive Bayes work well when combined with PCA but not when used together with ReliefF. These results make sense since both classifiers assume independent features and PCA just extracts uncorrelated features. However, any kind of independence is derived from the attributes chosen by ReliefF. Finally, as expected, the classification accuracies of nonlinear SVMs (with RBF kernel) are very poor, either in the validation or test partition. As discussed in Section 3.3, it is likely that the model complexity of RBF SVMs leads to overfitting and thus, the algorithm does not generalize well to unseen patterns [40]. In order to analyze the generalization capability of the best combinations in Figs. 6 and 7 as well as to compare them, Fig. 8 shows the classification accuracy (\%) in the validation and test sets for ReliefF and PCA when combined with both linear SVM and LDA. The difference between the validation and test errors is low, which means that the models are not incurring in overfitting.

Regarding the comparison between ReliefF + Linear SVM and PCA + LDA, both approaches provide good identification rates, being slightly better the combination of ReliefF + Linear SVM. Additionally, ReliefF + Linear SVM (i) is able to achieve an identification rate of $84.34 \%$ with only 60 features while PCA + LDA has not competitive results for this number of features, and (ii) favors the interpretability of the final model since ReliefF gives as a result a subset of the original features whereas PCA generates new variables from linear combinations of the original ones. Nevertheless, the good performance of PCA + LDA should not be dismissed. In fact, one of the advantages of this model with respect to ReliefF + Linear SVM is that we can depict its decision boundaries as shown in Fig. 9. When used for multi-class classification tasks, the classes are divided into two groups and the binary LDA is applied. Typically, two strategies can be implemented to generate such partitions. On the one hand, the "one against the rest" approach considers that points from one of the classes form one group and the points from the remaining classes configure the other group [8]. On the other hand, the "one against one" methodology generates a new classifier for each possible pair of classes [30]. The latter approach was the one adopted in this work and thus, one classifier was obtained for each pair of subjects (Sx vs Sy); that is, there are $\left(\begin{array}{c}13 \\ 2\end{array}\right)=78$ pairwise binary classifiers. This way, Fig. 9 shows the decision boundary of each binary classifier in one of the training/ test partitions of our evaluation process (see Fig. 4) as well as the points associated with these partitions. The good generalization

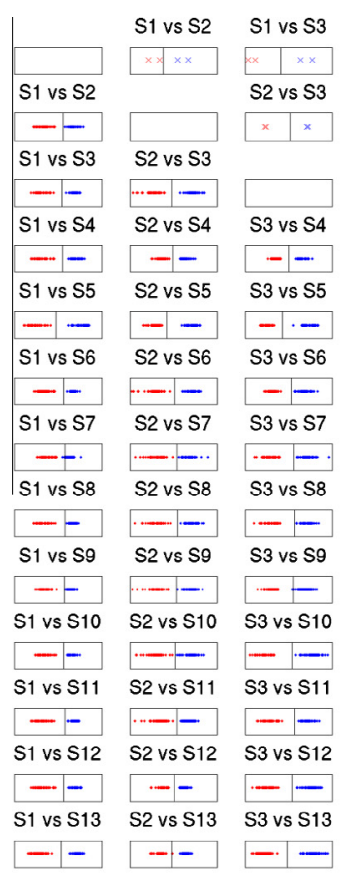

- Training points class 1

- Training points class 2
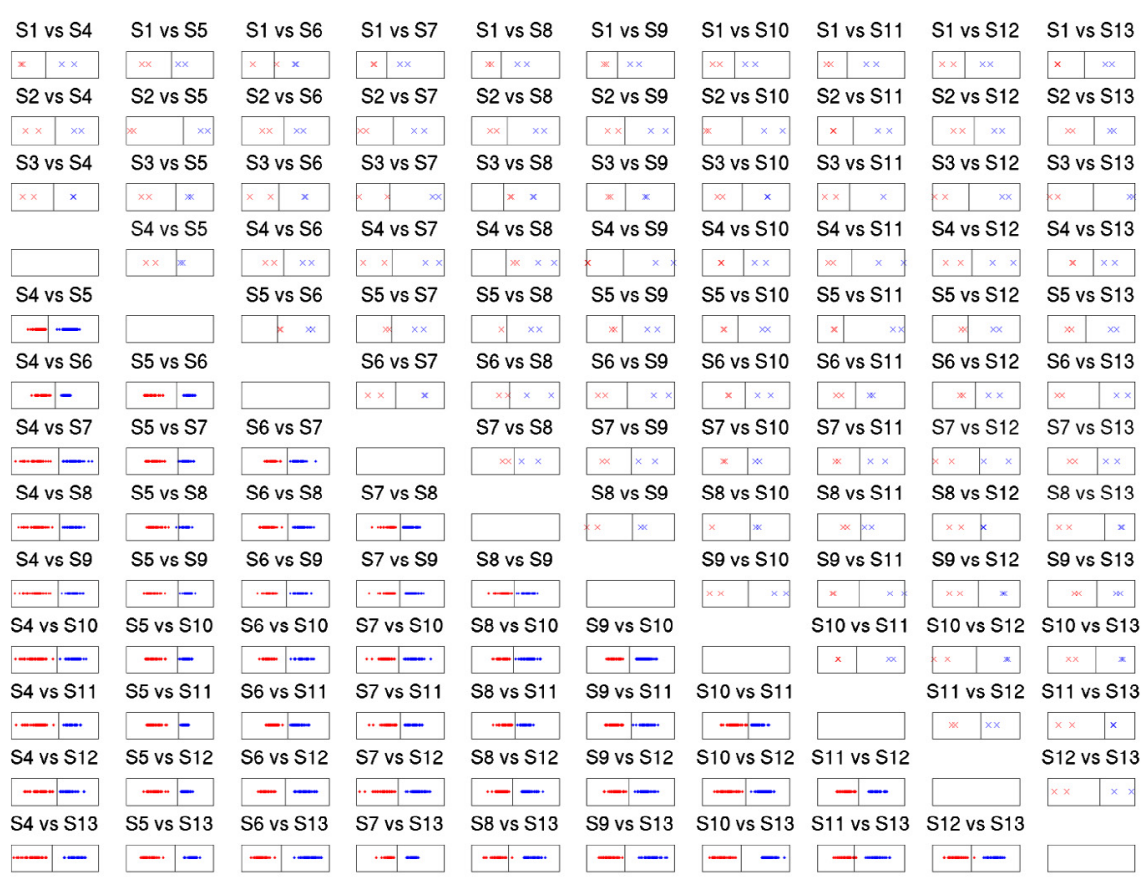

Test points class 1

Test points class 2

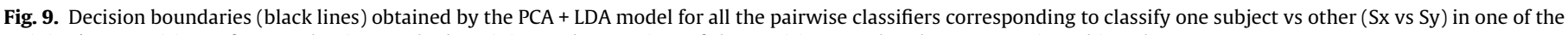
training/test partitions of our evaluation method. Training and test points of the partition are also shown. Best viewed in color. 

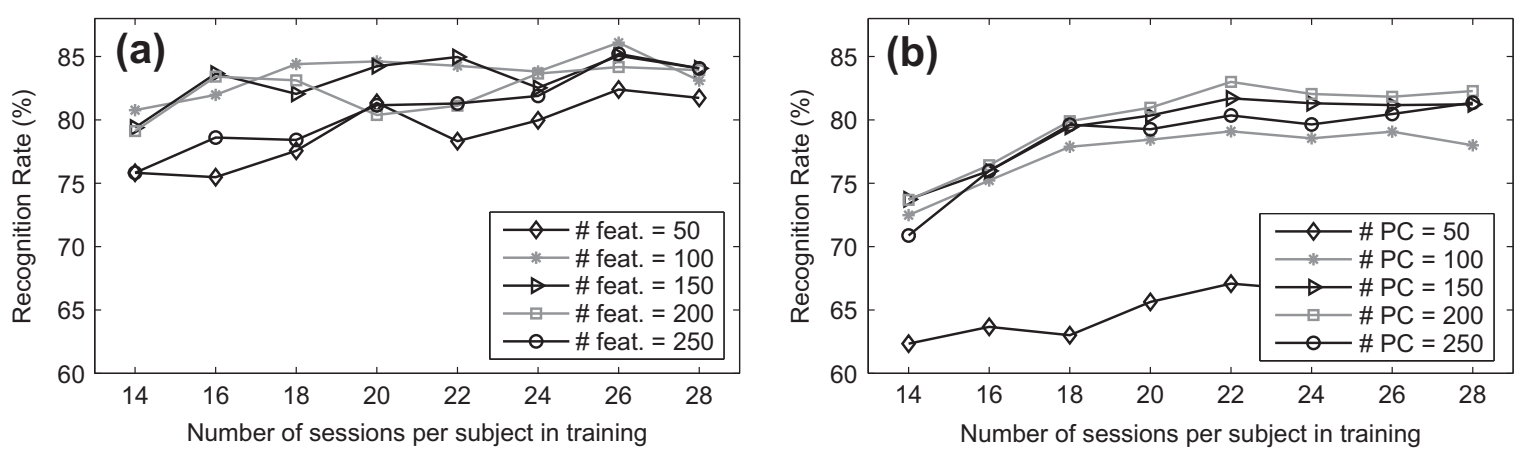

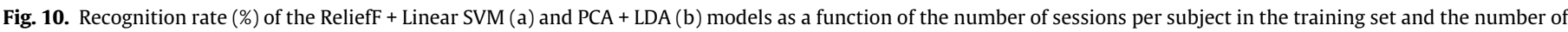
features (\#feat.) or principal components (\#PC), respectively.

of PCA + LDA is corroborated in this figure, being the test points well classified in most of the cases.

Finally, we would like to show the appropriateness of using our LOO-based evaluation strategy by analyzing the effect in the performance of varying the number of training sessions per subject for ReliefF + Linear SVM (Fig. 10a) and PCA + LDA (Fig. 10b). In the abscissa axis the number of sessions used in the training set is represented whereas the ordinate axis shows the recognition rate of the system. Each curve corresponds to the average recognition rate (\%) in test using a different number of features or principal components. It can be observed that in all cases the performance tends to improve as the number of sessions increases. The reason for this relation might be the high variability present in the body odor which makes it necessary to consider a large number of training samples in order to generate robust statistical models. Therefore, the methodology described in Section 4 is advisable as it is focused on maximizing the number of training samples.

\section{Conclusions}

This paper analyses the feasibility of using the body odor as a biometric identifier using a machine learning framework. Although the best classifier reaches an average recognition rate over $85 \%$ when identifying 13 subjects, these results are not comparable to other well-known biometric methods such as fingerprint or iris. Therefore, the identification based on the body odor should be complemented with other biometric techniques in order to increase its effectiveness. Nevertheless, these medium results are very promising since they reveal that there is actually discriminatory information in the body odor.

Due to the fact that this problem requires analyzing a large number of features (752 mass intervals) using a reduced number of samples (13 subjects $\times 28$ sessions), different dimensionality reduction approaches were tested together with different classifiers namely, k Nearest Neighbors (k-NN), Linear Discriminant Analysis (LDA), Logistic Regression, Naive Bayes and linear Support Vector Machines (SVMs). The incorporation of a dimensionality reduction phase not only reduced the computational cost of the identification system but also improved its effectiveness. More precisely, a filter feature selection method called ReliefF combined with a linear SVM yielded the best identification rate (85.03\%) with 160 features and it has demonstrated good generalization properties. A wrapper feature selection method achieved high recognition rates in the validation data but the results obtained with the testing data were degraded, questioning the generalization capacity of this model. Finally, Principal Component Analysis (PCA) was considered for feature extraction. PCA reached recognition rates over $82 \%$ when combined with LDA whereas providing good generalization results. However, it needed more variables (190 principal components) than the ReliefF approach.

In conclusion, this work gives evidence of the presence of distinctive information in the body odor by achieving recognition rates over $85 \%$ and outperforming the effectiveness of a random classifier in 11 times. Nevertheless, the odor sensor is still in a development phase and it is expected that further improvements lead to consider odor as a new biometric feature capable of competing with the most successful biometric techniques.

\section{Acknowledgements}

The authors thank the anonymous reviewers for their comments that help improve the manuscript. This work has been partially supported by Ministerio de Industria, Turismo y Comercio (Spain) under the Grant TSI-020100-2009-44.

\section{References}

[1] M. Beckmann, D. Parker, D.P. Enot, E. Duval, J. Draper, High-throughput, nontargeted metabolite fingerprinting using nominal mass flow injection electrospray mass spectrometry, Nat. Protocols 3 (2008) 486-504.

[2] U. Bernier, D. Kline, D. Barnard, C. Schreck, R. Yost, Analysis of human skin emanations by gas chromatography/mass spectrometry. 2. Identification of volatile compounds that are candidate attractants for the yellow fever mosquito (Aedes aegypti), Anal. Chem. 72 (2000) 747-756.

[3] U. Bernier, D. Kline, C. Schreck, R. Yost, D. Barnard, Chemical analysis of human skin emanations: comparison of volatiles from humans that differ in attraction of Aedes aegypti (Diptera: Culicidae), J. Am. Mosq. Control Assoc. 18 (2002) 186-195.

[4] C.M. Bishop, Pattern Recognition and Machine Learning, Springer, New York, 2006.

[5] S. Buratti, S. Benedetti, M. Scampicchio, E. Pangerod, Characterization and classification of Italian Barbera wines by using an electronic nose and an amperometric electronic tongue, Anal. Chim. Acta 525 (2004) 133-139.

[6] G.C. Cawley, N.L. Talbot, On over-fitting in model selection and subsequent selection bias in performance evaluation, J. Mach. Learn. Res. 99 (2010) 20792107.

[7] D. Chen, J. Haviland-Jones, Human olfactory communication of emotion, Percept. Motor Skill 91 (2000) 771-781.

[8] K. Crammer, Y. Singer, On the learnability and design of output codes for multiclass problems, Mach. Learn. 47 (2002) 201-233.

[9] A.M. Curran, P.A. Prada, K.G. Furton, The differentiation of the volatile organic signatures of individuals through SPME-GC/MS of characteristic human scent compounds, J. Forensic. Sci. 55 (2010) 50-57.

[10] A.M. Curran, S.I. Rabin, P.A. Prada, K.G. Furton, Comparison of the volatile organic compounds present in human odor using SPME-GC/MS, J. Chem. Ecol. 31 (2005) 1607-1619.

[11] A. D’Amico, R. Bono, G. Pennazza, M. Santonico, G. Mantini, M. Bernabei, M. Zarlenga, C. Roscioni, E. Martinelli, R. Paolesse, et al., Identification of melanoma with a gas sensor array, Skin Res. Technol. 14 (2008) 226-236.

[12] S.J. Dixon, Y. Xu, R.G. Brereton, H.A. Soini, M.V. Novotny, E. Oberzaucher, K. Grammer, D.J. Penn, Pattern recognition of gas chromatography mass spectrometry of human volatiles in sweat to distinguish the sex of subjects and determine potential discriminatory marker peaks, Chemometr. Intell. Lab. 87 (2007).

[13] C. Doyle, The secret cloud that surrounds us, Fam Health 32 (1970).

[14] R.O. Duda, P.E. Hart, D.G. Stork, Pattern Classification, Wiley, New York, 2001 
[15] J. Fonollosa, A. Vergara, R. Huerta, Algorithmic mitigation of sensor failure: is sensor replacement really necessary?, Sens Actuators, B 183 (2013) 211-221

[16] M. Gallagher, C.J. Wysocki, J.J. Leyden, A.I. Spielman, X. Sun, G. Preti, Analyses of volatile organic compounds from human skin, Brit. J. Dermatol. 159 (2008) 780-791.

[17] M.D. Gibbs, Biometrics: body odor authentication perception and acceptance, SIGCAS Comput. Soc. 40 (2010) 16-24.

[18] R. Gosangi, R. Gutierrez-Osuna, Active temperature programming for metaloxide chemoresistors, IEEE Sens. J. 10 (2010) 1075-1082.

[19] P. Groscurth, Anatomy of sweat glands, Cur. Probl. Dermatol. 30 (2002) 1-9.

[20] R. Gutierrez-Osuna, Pattern analysis for machine olfaction: a review, IEEE Sens. J. 2 (2002) 189-202.

[21] R. Gutierrez-Osuna, A. Gutierrez-Galvez, N. Powar, Transient response analysis for temperature-modulated chemoresistors, Sens. Actuators, B 93 (2003) 57-66.

[22] I. Guyon, S. Gunn, M. Nikravesh, L. Zadeh (Eds.), Feature Extraction: Foundations and Applications, first ed., Springer, 2006.

[23] T. Hastie, R. Tibshirani, J.J.H. Friedman, The Elements of Statistical Learning, vol. 1, Springer, New York, 2001.

[24] J. Havlicek, P. Lenochova, Environmental effects on human body odour, Chem. Signal 11 (2008) 199-210.

[25] R. Heckle, A. Patrick, A. Ozok, Perception and acceptance of fingerprint biometric technology, in: Proceedings of the 3rd Symposium on Usable Privacy and Security, ACM, 2007, p. 154.

[26] S. Jenkins, Can Police Dogs Identify Criminal Suspects by Smell? Using Experiments to Test Hypotheses about Animal Behavior, Oxford University Press, New York, 2004.

[27] I. Jolliffe, Principal Component Analysis, Springer Verlag, 2002.

[28] A. Juels, D. Molnar, D. Wagner, Security and privacy issues in e-passports, in: Security and Privacy for Emerging Areas in Communications Networks (SecureComm), 2005, pp. 74-88.

[29] K. Kira, L.A. Rendell, A practical approach to feature selection, in: Proceedings of the Ninth International Workshop on Machine Learning, Morgan Kaufmann Publishers Inc., San Francisco, CA, USA, 1992, pp. 249-256.

[30] S. Knerr, L. Personnaz, G. Dreyfus, Single-layer learning revisited: a stepwise procedure for building and training a neural network, in: Neurocomputing: Algorithms, Architectures and Applications, NATO ASI Series, vol. F68, Springer-Verlag, 1990, pp. 41-50.

[31] F. Kuhn, A. Natsch, Body odour of monozygotic human twins: a common pattern of odorant carboxylic acids released by a bacterial aminoacylase from axilla secretions contributing to an inherited body odour type, J. Roy. Soc. Interface 6 (2009) 377.

[32] P. Lenochova, J. Havlicek, Human body odour individuality, Chem. Signal 11 (2008) 189-198.

[33] R.H. Lilien, H. Farid, B.R. Donald, Probabilistic disease classification of expression-dependent proteomic data from mass spectrometry of human serum, J. Comput. Biol. 10 (2003) 925-946.

[34] Y. Liu, Feature extraction and dimensionality reduction for mass spectrometry data, Comput. Biol. Med. 39 (2009) 818-823.

[35] D. Maltoni, D. Maio, A. Jain, S. Prabhakar, Handbook of Fingerprint Recognition, Springer-Verlag New York Inc., 2009.

[36] S. Marco, A. Gutierrez-Galvez, Signal and data processing for machine olfaction and chemical sensing: a review, IEEE Sens. J. 12 (2012) 3189-3214.

[37] P. Martínez-Lozano, J. de la Mora, On-line detection of human skin vapors, J. Am. Soc. Mass Spectr. 20 (2009) 1060-1063.

[38] P. Miketova, C. Abbas-Hawks, K.J. Voorhees, T.L. Hadfield, Microorganism Gram-type differentiation of whole cells based on pyrolysis high-resolution mass spectrometry data, J. Anal. Appl. Pyrol. 67 (2003) 109-122.

[39] E. Mostacci, C. Truntzer, H. Cardot, P. Ducoroy, Multivariate denoising methods combining wavelets and principal component analysis for mass spectrometry data, Proteomics 10 (2010) 2564-2572.

[40] K.R. Müller, S. Mika, G. Rätsch, K. Tsuda, B. Schölkopf, An introduction to kernelbased learning algorithms, IEEE Trans. Neural Netw. 12 (2001) 181-201.

[41] S. Munk, P. Munch, L. Stahnke, J. Adler-Nissen, P. Schierberle, Primary odorants of laundry soiled with sweat/sebum: influence of lipase on the odour profile, J. Surfactants Deterg. 3 (2000) 505-515.
[42] A. Natsch, H. Gfeller, P. Gygax, J. Schmid, G. Acuna, A specific bacterial aminoacylase cleaves odorant precursors secreted in the human axilla, J. Biol. Chem. 278 (2003) 5718-5727.

[43] N. Nicolaides, Skin lipids: their biochemical uniqueness, Science 186 (1974) 19-26.

[44] D.J. Penn, E. Oberzaucher, K. Grammer, G. Fischer, H.A. Soini, D. Wiesler, M.V. Novotny, S.J. Dixon, Y. Xu, R.G. Brereton, Individual and gender fingerprints in human body odour, J. Roy. Soc. Interface 4 (2007) 331.

[45] J. Prados, A. Kalousis, J.C. Sanchez, L. Allard, O. Carrette, M. Hilario, Mining mass spectra for diagnosis and biomarker discovery of cerebral accidents, Proteomics 4 (2004) 2320-2332+.

[46] Y. Qu, B.I. Adam, M. Thornquist, J.D. Potter, M.L. Thompson, Y. Yasui, J. Davis, P.F. Schellhammer, L. Cazares, M. Clements, et al., Data reduction using a discrete wavelet transform in discriminant analysis of very high dimensionality data, Biometrics 59 (2003) 143-151.

[47] S. Raychaudhuri, J.M. Stuart, Russ B. Altman, Principal components analysis to summarize microarray experiments: application to sporulation time series, in: Pacific Symposium on Biocomputing, 2000, pp. 452-463.

[48] I. Rodriguez-Lujan, C. Santa Cruz, R. Huerta, On the equivalence of kernel Fisher discriminant analysis and kernel quadratic programming feature selection, Pattern Recognit. Lett. 32 (2011) 1567-1571.

[49] Y. Saeys, I. Inza, P. Larrañaga, A review of feature selection techniques in bioinformatics, Bioinformatics 23 (2007) 2507-2517.

[50] S. Sastry, K. Buck, J. Janak, M. Dressler, G. Preti, Volatiles emitted by humans, Biochem. Appl. Mass Spectrom (Supplement) (1980) 1085-1129.

[51] G.A.A. Schoon, Scent identification lineups by dogs (Canis familiaris): experimental design and forensic application, Appl. Anim. Behav. Sci. 49 (1996).

[52] B.A. Sommerville, D. Gee, Research on body odours: new prospects for combating crime?, IC.P.R. (1987) 18-22.

[53] R.A. Stockham, Bloodhounds and bombing investigations, Detonator 30 (2003) 35-37.

[54] M. Turk, A. Pentland, Face recognition using eigenfaces, in: Proceedings CVPR '91., IEEE Computer Society Conference on Computer Vision and Pattern Recognition, 1991, pp. 586-591.

[55] V. Vapnik, A. Chervonenkis, Theory of Pattern Recognition, Nauka, 1974 (in Russian).

[56] G. Vidal-de-Miguel, A. Herrero, Secondary electrospray ionization of complex vapor mixtures. theoretical and experimental approach, J. Am. Soc. Mass Spectr. 23 (2012) 1085-1096.

[57] G. Vidal-de-Miguel, M. Macía, P. Pinacho, J. Blanco, Low-sample flow secondary electrospray ionization: improving vapor ionization efficiency, Anal. Chem. 84 (2012) 8475-8479.

[58] M. Wagner, D.G. Castner, Characterization of adsorbed protein films by timeof-flight secondary ion mass spectrometry with principal component analysis, Langmuir 17 (2001) 4649-4660.

[59] X. Wang, M. Ye, C. Duanmu, Classification of data from electronic nose using relevance vector machines, Sens. Actuators, B 140 (2009) 143-148.

[60] C.J. Wysocki, G. Preti, Facts, fallacies, fears, and frustrations with human pheromones, Anat. Rec. A Discov. Mol. Cell. Evol. Biol. 281 (2004) $1201-$ 1211.

[61] X. Zeng, J. Leyden, H. Lawley, K. Sawano, I. Nohara, G. Preti, Analysis of characteristic odors from human male axillae, J. Chem. Ecol. 17 (1991) 14691492.

[62] X. Zeng, J. Leyden, A. Spielman, G. Preti, Analysis of characteristic human female axillary odors: qualitative comparison to males, J. Chem. Ecol. 22 (1996) 237-257.

[63] Z.M. Zhang, J.J. Cai, G.H. Ruan, G.K. Li, The study of fingerprint characteristics of the emanations from human arm skin using the original sampling system by SPME-GC/MS, J. Chromatogr. B 822 (2005).

[64] W. Zhao, R. Chellappa, A. Krishnaswamy, Discriminant analysis of principal components for face recognition, in: Proceedings. Third IEEE International Conference on Automatic Face and Gesture Recognition, 1998, pp. 336-341.

[65] Y. Zhu, T. Tan, Y. Wang, Biometric personal identification based on handwriting, Int. C. Pattern Recog. 2 (2000) 2797. 